\title{
A C TYPE DIPOLE WITH IMBEDDED WINDINGS TO IMPROVE THE FIELD DISTRIBUTION
}

\author{
P.Yuan, Y.He, L.Z.Ma, B.Zhang, W.J.Liu, X.F.Han, H.Xiong, W.J.Wang, J.Wu, R.R.He, J.W.Xia, \\ and W.L.Zhan, W.B.Wei, IMP, CAS, Lanzhou, P.R.China
}

\begin{abstract}
A method is described in this paper, which introduces some slots in the $\mathrm{C}$ type dipole pole and some conductors can be put inside these slots. These conductors can control the flow of the magnetic flux by adjusting its trim current, thus that can improve the magnetic field distribution at the median plane of this dipole. The effect of the trim current for low field was investigated using OPERA-2D and 3D, some results will be present in this paper. Furthermore, the fabricating technology of this $\mathrm{C}$ type dipole and some magnetic field measurement results will be discussed in this paper as well.
\end{abstract}

\section{INTRODUCTION}

HIRFL-CSR, a new accelerator project at the Heavy Ion Research Facility in Lanzhou (HIRFL), is a multipurpose Cooling Storage Ring system that consists of a main ring $(\mathrm{CSRm})$ and an experimental ring (CSRe)[1] The heavy ion beams with the energy range of 10 50 MeV/u from the cyclotron will be accumulated, cooled and accelerated to the high energy range of $100 \sim 600 \mathrm{MeV} / \mathrm{u}$ in the main ring $(\mathrm{CSRm})$, extracted fast to produce radioactive ion beams (RIB) or highly charged heavy ions. The experimental ring (CSRe) can accept the secondary beams (RIB or highly charged heavy ions) for many internaltarget experiments or high precision spectroscopy with beam cooling.

Because it is required to install detectors of charge exchange beside vacuum chamber along the main dipoles of CSRe, the dipoles have got to be designed with the Ctype and its the good field region is wide to $220 \mathrm{~mm}$. For it was decided that no reference and prototype magnets were to be constructed, after OPERA code calculation, the bending magnets in the injection beam line from SSC to CSRm were selected as CSRe dipoles fabrication' R\&D.

\section{THE SPECIAL C TYPE DIPLOE CALCULATION AND FABRICATION}

\subsection{Design calculation}

Main parameters of the $\mathrm{C}$ type dipole the injection beam line are given at table1. A method to obtain good field homogeneity was tried in this $\mathrm{C}$ type dipole, similar the idea suggested by K.Halbach for $\mathrm{H}$ type dipole[2].

Since the flux in the $\mathrm{C}$ type dipole iron has different path lengths, and with finite permeability it will have a lower B field in the outside gap than in the inside gap, So the field homogeneity will become sloped and form an unwanted gradient when the exciting current changed. However, while two slots were punched on the dipole pole and a trim conductor was installed inside the slots separately, the situation of the field distribution will be improved by adjusting trim conductors' current, shown in Fig.1.

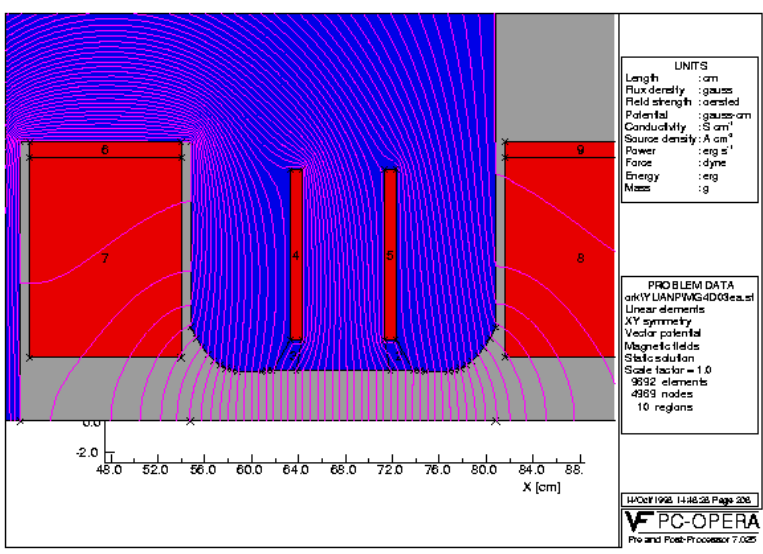

Fig.1 Magnetic flux distribution in the special $\mathrm{C}$ type dipole, 4 and 5 are trim conductors.

Table1 Main parameters of the $\mathrm{C}$ type dipole.

\begin{tabular}{|l|l|}
\hline & Dipole \\
\hline Number & 2 \\
\hline Max. Field $\quad(\mathrm{T})$ & 1.53 \\
\hline Bending radius $\quad(\mathrm{m})$ & 2.0 \\
\hline Bending angle $\quad($ degree $)$ & 60 \\
\hline Effective length $(\mathrm{m})$ & 2.0 \\
\hline Effective aperture $\quad\left(\mathrm{mm}^{2}\right)$ & $100 \times 55$ \\
\hline Air gap $\quad(\mathrm{mm})$ & 65.0 \\
\hline Field uniformity $\quad \pm 1.0 \times 10^{-3}$ \\
\hline Coil turns/pole & 36 \\
\hline Current $\quad(\mathrm{A})$ & 1250 \\
\hline Resistance $\quad(\mathrm{Ohm})$ & 0.025 \\
\hline Inductance $\quad(\mathrm{H})$ & 0.058 \\
\hline \multicolumn{2}{|l}{} \\
\hline
\end{tabular}

In Fig.2, the field homogeneity was compared with trim conductor and without it in the good field region at different field level. It is very clear the trim conductor's adjusting effect is very obviously for the lower field.

After OPERA-2D calculation, in order to check the field uniformity variation influenced by the fringe field and permeability saturation in the end of the magnet, TOSCA was used to calculate the special $\mathrm{C}$ type dipole with the same dimension size and only adjusting the iron shim on the pole surface. Its mesh model was shown in the Fig.3. 


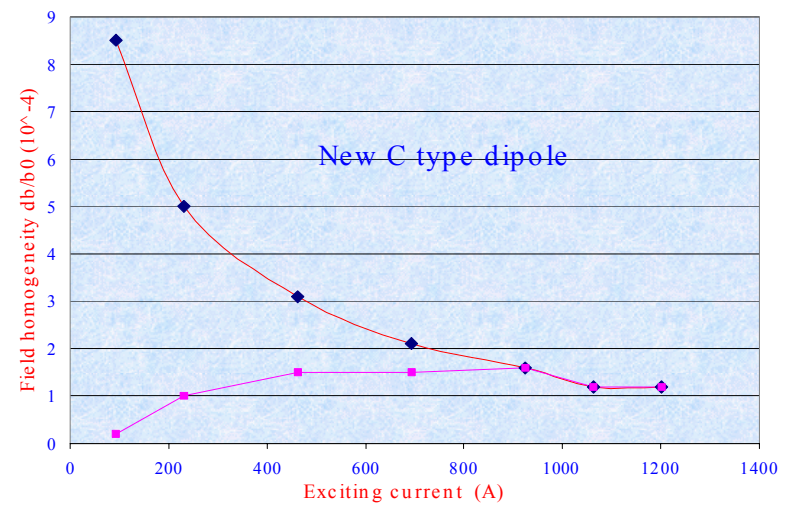

Fig.2 Comparison of field homogeneity in the good field region with trim conductors and without trim conductor.

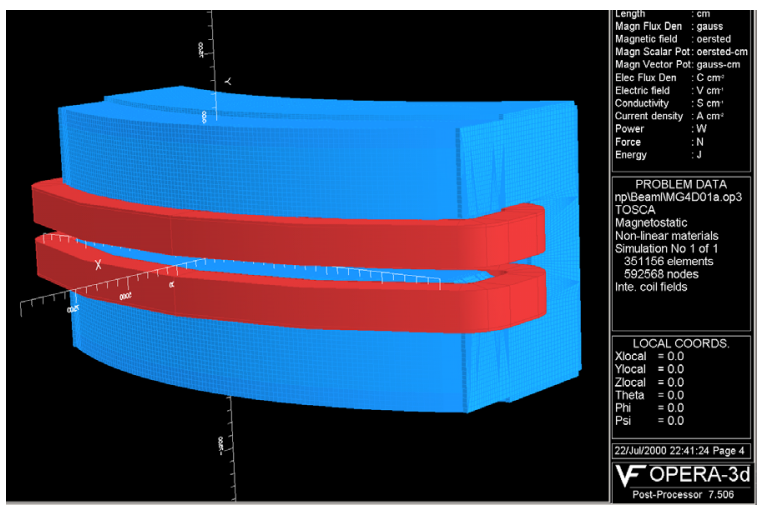

Fig.3 3D mesh of the special C type dipole model

\subsection{Magnet fabrication}

The magnet iron core was made of $0.5 \mathrm{~mm}$ thick high induction cold rolled isotropy electro-technical steel sheets J23-50 (made in Wuhan Iron and Steel Company). Coils were made of T2 copper hollow conductor and insulated with polyimide stick tape and polyimide micrapowder tape pre-impregnated with epoxy resin.

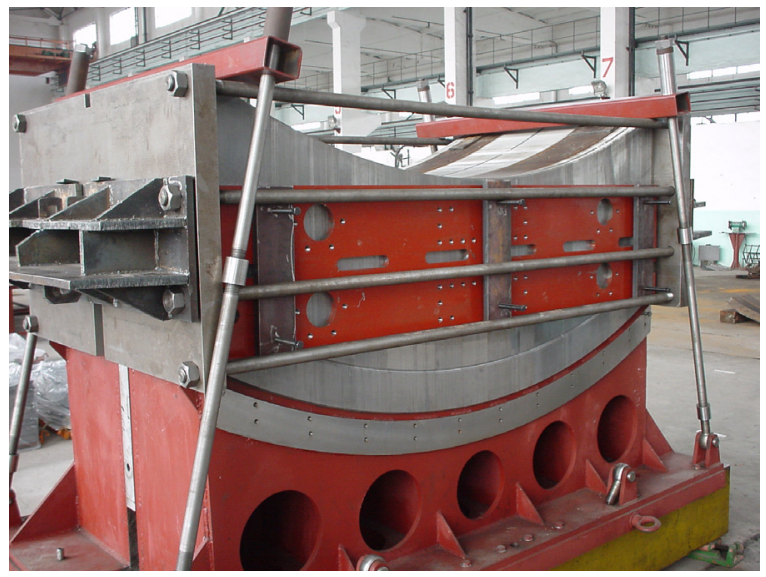

Fig.4 The special $\mathrm{C}$ type dipole stacking on the tool table

The open mouth side of this $\mathrm{C}$ type dipole was taken as a reference arc surface and a reference guide was inserted into the gap. There have two $200 \mathrm{~mm}$ endplates also made of the same laminate sheets in the two side to enhance its mechanical strength. Because the dipole has a larger bending angle and smaller radius, it is very difficulty to stack and keep its arc shape of laminate body connecting to the reference arc surface. So two endplates were glued and stacked in the same stacking table, see the Fig.4. Four side plates and two upper plates and two lower plates were welded altogether with laminate sheets in a spiral sequence step by step. After welding and painting, the $60^{\circ}$ $\mathrm{C}$ type dipole prototype was shown in the Fig.5.

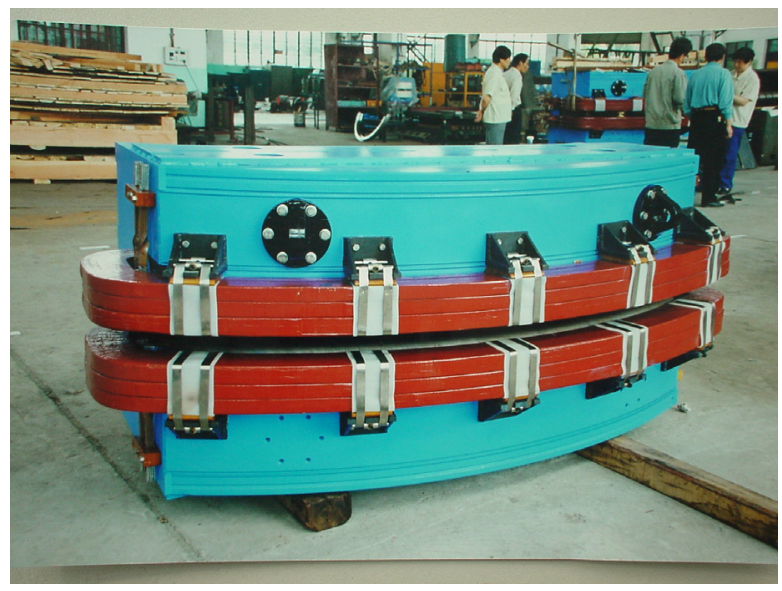

Fig.5 The C type dipole of CSRm injection beam line.

\section{FIELD MEASUREMENT RESULTS}

Fig.6 shows the actuating curve of the $\mathrm{C}$ type dipole, the square marks are the calculated data by TOSCA and connecting to a curve. The four star marks are the measured data by the hall probe, and it's more correctly coincident with TOSCA calculation's results. The magnetic field transversal distribution at good field region is shown in the Fig.7, that's also measured by the hall probe, the lower curve is without trim conductor current and the upper curve is with only $2 \mathrm{~A}$ current for the outside trim conductor.

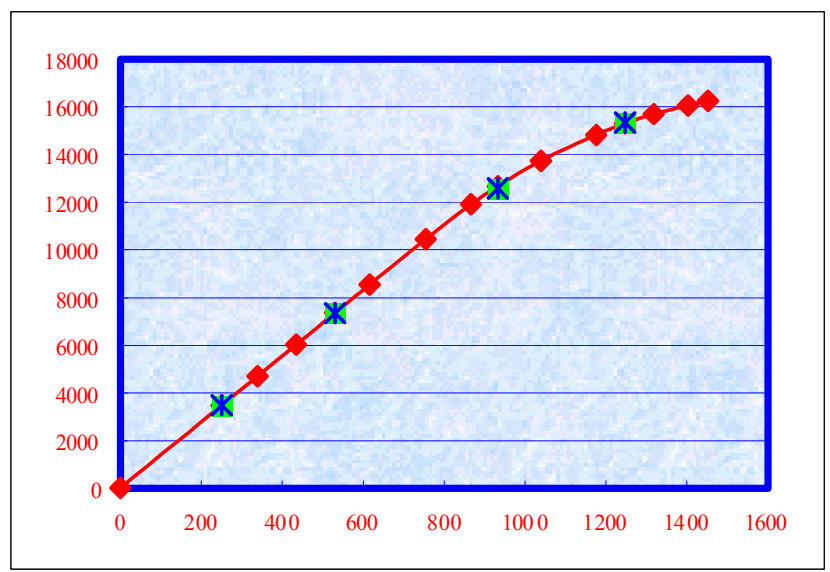

Fig.6 Actuating curve of the $\mathrm{C}$ type dipole 


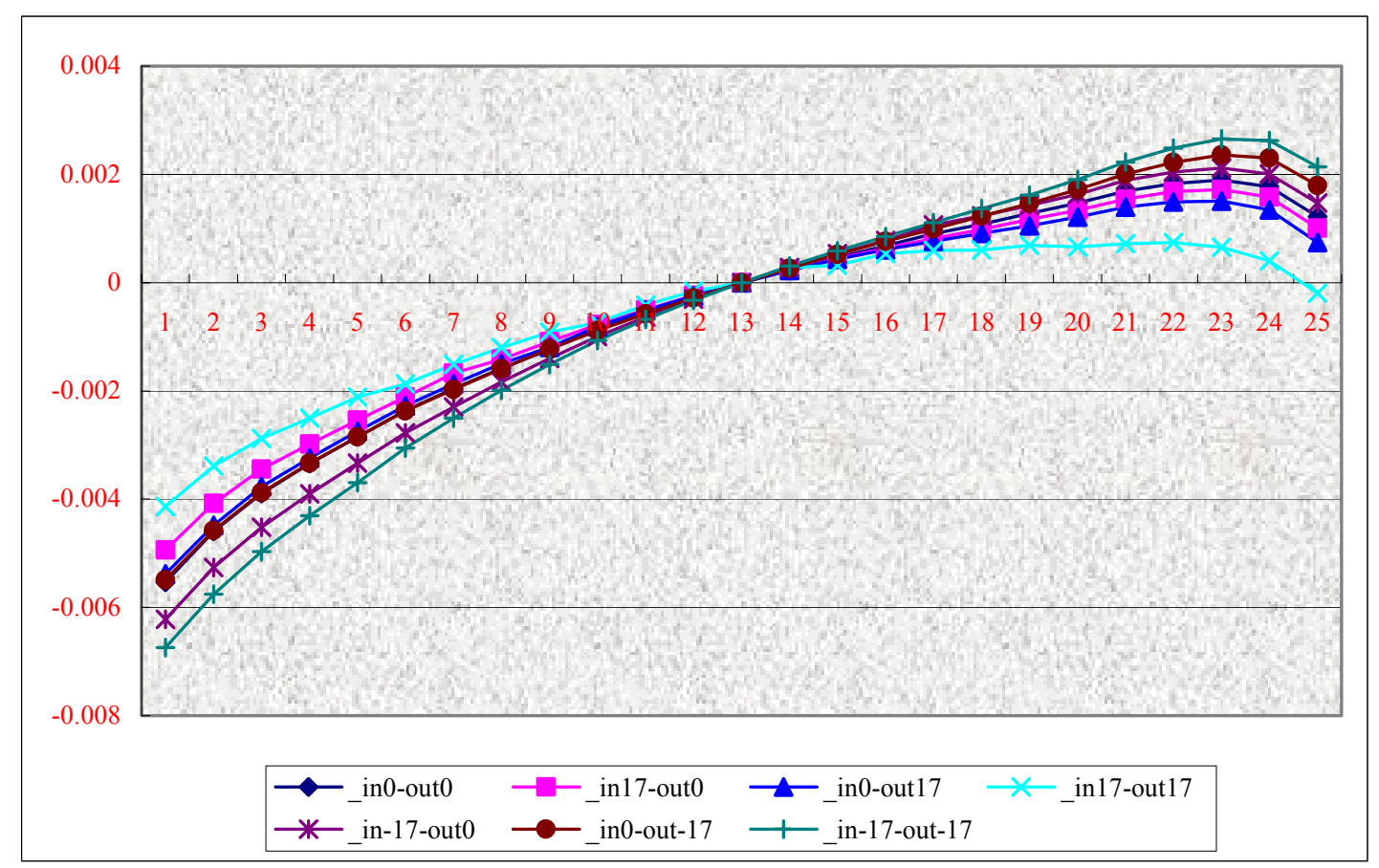

Figure8: The influence of the trim conductors' current on the integral magnetic field distribution at good field region

In order to measure the integral field distribution at good field region, a long and bending integral coil was made with G10 material and winding with $0.1 \mathrm{~mm}$ wires, this long integral coil's bending radius is the same as the $60^{\circ} \mathrm{C}$ type dipole and its effective region length is about $3 \mathrm{~m}$. The measured results at low filed of about $2400 \mathrm{Gs}$ are shown in the Figure8, the transversal axis is displayed the good field region from $-60 \mathrm{~mm}$ to $60 \mathrm{~mm}$, and from the plus mark line to the product sign mark line the trim conductor current changed from $-17 \mathrm{~A}$ to $17 \mathrm{~A}$ for inside and outside trim conductors respectively. That's clear the integral field distribution become much better changed by the trim conductor current, the outside peak is lowering and the inside is going up step by step by increasing the current from-17A to 17A.

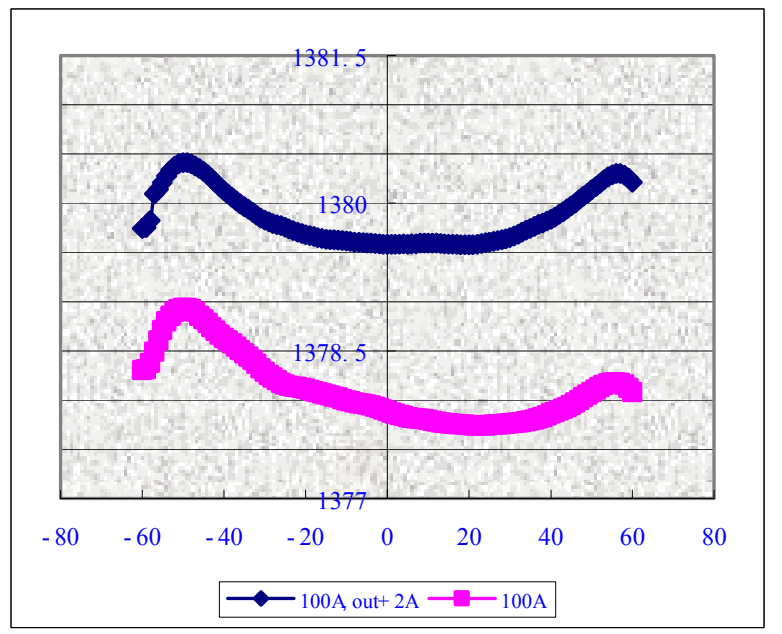

Fig.7 The field transversal distribution at 1300Gs
For the high field, only hall probe point measurement was got at good field region along transversal axis. The trim conductor current's action is obviously too, shown in the Fig.9, the lowest line is without trim current, the middle line is with $100 \mathrm{~A}$ trim current for inner conductor, and the highest line is with 200A for this conductor.

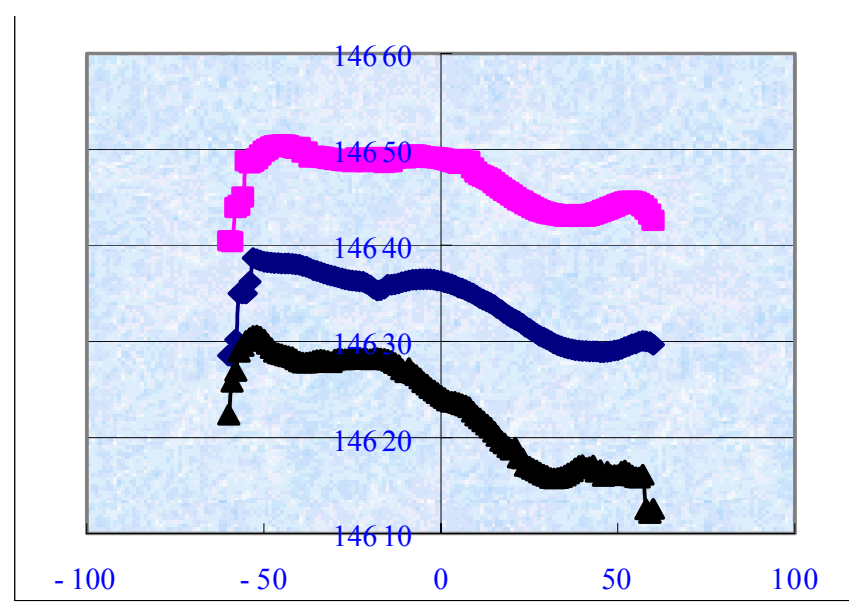

Fig.9 The field distribution improved by inner trim conductor at $14600 \mathrm{Gs}$

\section{REFERENCES}

[1] J.W.Xia, et al., "HIRFL Status and HIRFL-CSR Project in Lanzhou", APAC'98, KEK, June 1998

[2] K.Halbach, "Field Correction Windings for Iron Magnets", N.I.M., 107(1973), 515-528 